\title{
Acquisition, extinction, and reacquisition of a conditional discrimination to avoid shock by goldfish
}

\author{
D. J. ZERBOLIO, JR. \\ University of Missouri, St. Louis, Missouri
}

\begin{abstract}
Goldfish, trained in the shuttlebox to avoid shock, were tested for the acquisition and extinction of a color-matching or a color-oddity conditional discrimination choice response, and then tested for reacquisition. Extinction affected the accuracy of the choice response but not the number of trials with response (response strength). Half of all groups were extinguished with changed signal colors, and half had the same signal colors experienced in acquisition. All groups had the same signal colors in reacquisition that they had experienced in acquisition. Changedsignal oddity groups decremented slightly faster than same-signal oddity groups, providing some support for a generalization decrement interpretation, but same- and changed-signal matching groups did not differ. All groups extinguished on the choice response by the end of extinction. All matching groups, and all oddity groups, regardless of their respective signal colors in extinction, reacquired at the same rate, and faster than in acquisition. These results imply conceptual generalization of extinction effects. Matching-trained groups were found to be slightly superior to oddity-trained groups in both acquisition and reacquisition. Comparisons of these results to positive reinforcement conditional discrimination extinction work, including some procedural suggestions, are made.
\end{abstract}

With a conditional discrimination procedure in the shuttlebox, goldfish learned to choose the matching or odd signal color to avoid shock (Zerbolio \& Royalty, 1983). In a transfer test, goldfish with new signal colors continued to conditionally discriminate as well as goldfish tested with their original color, as long as the criterion contingency was the same (matching or oddity) in both original learning and transfer testing. If the criterion contingency was shifted in transfer testing (matching to oddity or oddity to matching), massive negative transfer effects occurred, regardless of whether the transfer signal colors were the same or changed from those in original learning. All of these observations are consistent with a conceptual learning interpretation, specifically, that goldfish learned about the relationship between colors in original learning (matching or oddity choice) and this relationship transferred to at least one other (new) color set. If the conceptual learning interpretation is valid, additional predictions can be made for extinction.

Suppose two groups are initially trained to choose the matching (or odd) color in acquisition. Next, one group has its choice response extinguished using the same signal colors as in acquisition, and the second group is extinguished with a new signal color set. If both groups are then tested in a reacquisition procedure with their original acquisition signal colors, a

The author's mailing address is: Department of Psychology, University of Missouri, St. Louis, MO 63121. comparison of their choice reacquisition performances is possible. If conceptual transfer occurs, both groups should have had their matching or oddity choice responses extinguished, regardless of the signal colors used in extinction, and therefore should reacquire identically. If conceptual transfer has not occurred, the group extinguished with new colors should reacquire faster.

Obviously, the key to testing this prediction is a procedure that extinguishes the accuracy of the matching/oddity choice response. Earlier studies, using pigeons, food reinforcement, and the traditional extinction procedure of turning off the food dispenser, have uniformly found substantial decrements in the strength of the keypeck response, but little if any decrement in the accuracy of the choice response (Cohen, 1969; Cumming, Berryman, Cohen, \& Lanson, 1967; Looney, Cohen, Brady, \& Cohen, 1977; Lydersen \& Cheney, 1973). Clearly, an extinction procedure that does not cause the accuracy of the choice response to diminish would be inadequate for the present purposes. Fortunately, Zerbolio and Royalty (1982) have developed what may be the appropriate procedure. Following the acquisition of a simple color choice discrimination, the contingency requiring the goldfish to differentially choose $\left(R_{c}\right)$ was suspended, but the contingency requiring the goldfish to initially respond $\left(\mathbf{R}_{1}\right)$ was retained. Goldfish continued to respond on the same or a greater number of trials as they had in acquisition (TwR), but their color choice accuracy $\left(R_{c}\right)$ dimin- 
ished to chance performance levels. The aim of the present study is to adapt this extinction procedure to the conditional discrimination problem, and then use it to test for the conceptual transfer of extinction.

\section{METHOD}

\section{Subjects}

Twenty-four 5-6-cm-long goldfish, obtained from Ozark Fisheries and housed in $7.5 \times 11.5 \times 12.5 \mathrm{~cm}$ deep individual holding tanks, served as subjects. Water in the holding tanks was well filtered and aerated at all times. About $25 \%$ of the water was removed daily, by siphoning, which also removed debris, and replaced with fresh water. Tanks were emptied, scrubbed, and refilled weekly. Temperature $\left(21 \pm .1^{\circ} \mathrm{C}\right)$ and $\mathrm{pH}(7 \pm .1)$ were held constant throughout the experiment. The fish were fed daily.

\section{Apparatus}

Four identical $29.2 \times 11.4 \times 11.4 \mathrm{~cm}$ deep aquatic shuttleboxes (tanks) were used (see Zerbolio \& Royalty, 1983). Each shuttlebox contained a sandblasted translucent hurdle, $6.35 \mathrm{~cm}$ high, with a 9 cm-long flat on top, and with 45-deg sloping ramps to deep wells in both ends. Water was maintained at $2 \mathrm{~cm}$ over the top of the hurdle at all times. Constant aeration was provided by inserts in the ends of the shuttletanks. During testing, all shuttletanks were covered with a clear plastic lid. Light sources for photocells, positioned at the ends of the center flat of the hurdle $9 \mathrm{~cm}$ apart and shining horizontally across the tank into photocells, were $2.5-\mathrm{V} \mathrm{dc}$ prefocused penlight bulbs, operated at $1.5 \mathrm{~V}$ ac. Shuttle activity was monitored by these photocells. All colored light signals were provided by $7-\mathrm{W} 110-\mathrm{V}$ ac Christmas tree lights. Four light fixtures were placed around each shuttletank and provided differential color illumination for the intertrial interval (ITI), the current trial standard, and response-contingent color illumination. ITI illumination was provided by a fixture placed on top of the tank during testing. The current-trial standard-color illumination was provided by a fixture centered underneath the tank, which cast light directly upward, illuminating the center hurdle through a 5 $\mathrm{cm}$-wide slit across the bottom of the tank. The rest of the bottom of the shuttletank was painted with an opaque flat black paint. An opaque black plastic baffle placed inside and across the center of the hurdle, served to visually isolate the color of the ends so that a fish in the deep well on one end could see the color of the end it occupied and the color of the center hurdle on that side, but could not see the color of the other end without moving to the top of the hurdle. The remaining two fixtures were located at the ends of the shuttletanks and provided response-contingent color illumination of the tank end-wall surfaces. Translucent $.16-\mathrm{cm}$ plastic plates were placed between each light source and the tank to diffuse illumination evenly. The red, green, blue, and yellow Christmas tree bulbs had measured spectral peaks of $620 \pm 4 \mathrm{~m} \mu, 517 \pm 2 \mathrm{~m} \mu, 474$ $\pm 2 \mathrm{~m} \mu$, and $286 \pm 2 \mathrm{~m} \mu$, respectively. Obvious brightness differences between different colored bulbs were adjusted by placing a diode in series with the 110-V ac power line for the red and yellow sources and using two bulbs for the green and blue sources. Luminances were measured following these adjustments using a Macbeth illuminometer, positioned $10 \mathrm{~cm}$ above the center hurdle, through water in the normally filled tank. Luminances of the center hurdle trial standard colors were measured through $2 \mathrm{~cm}$ of water, and for the end lights, using a reflecting mirror, through a total path of $15 \mathrm{~cm}$ of water. In general, measured luminances for the center-hurdle-trial standard-color sources were brighter than the end light sources. For the center hurdle, measured luminances for the red, blue, green, and yellow lights were 8-13, 5-10, 6-15, and 4-10 mL, respectively, and for the end light sources, 1-4, 1-2, $1-4$, and $1-4 \mathrm{~mL}$, respectively. Variability in luminance between lights of the same color was the same as that between lights of different color. The brightness of the white overhead ITI light was also attenuated with a series diode and measured $7 \mathrm{~mL}$. Shock, in 200 -msec pulses at $7 \mathrm{~V}$ ac $(.69 \mathrm{~V} / \mathrm{cm})$ was delivered by $28 \times$ $10.2 \mathrm{~cm} 22$-ga stainless steel plates affixed to the interior side walls of each shuttletank.

\section{Procedure}

The experiment was divided into three phases: an original acquisition phase ( 30 days), an extinction phase ( 30 days), and a reacquisition phase ( 30 days), for a total of 90 running days. The goldfish were run $\mathbf{4 0}$ trials a day, 5 days a week. They rested on weekends. Trials were separated by a variable 60 -sec ITI, ranging from 30 to $90 \mathrm{sec}$. Different illumination or colors indicated the ITI, trial start, current-trial standard color, and response-contingent color states to the animal. White overhead illumination indicated the ITI state. Termination of the white ITI state (to dark) indicated the start of a trial, which was followed $2 \mathrm{sec}$ later by the onset of current trial standard (differential color illumination) from underneath the hurdle. The specific color of a current trial standard was irregularly determined (Gellerman series) so that for each day's $\mathbf{4 0}$ trials, a random half were one color (e.g., red) and the remainder were the other color (e.g., green). The end lights remained dark until the animal made its first shuttle response $\left(R_{1}\right)$. A response was defined as the animal's leaving the deep end well occupied at trial onset and swimming over the top of the hurdle through the farthest photo beam. Breaking the near photo beam (the one closest to the animal at trial onset) did not constitute a response. The animals very rarely remained atop the hurdle during the ITI because the water was too shallow $(2 \mathrm{~cm})$. Following the first response $\left(\mathbf{R}_{1}\right)$ of the trial, the end light sources were illuminated. The color of illumination contingent on the first response was random (e.g., either red or green), and both ends were illuminated with the same color. Additional shuttle responses after the initial $\mathbf{R}_{1}$ response caused the color of the end lights to alternate with each response (e.g., red to green, or vice versa). During the trial period, the color illumination of the center hurdle trial standard remained constant, but the color of the end lights could be changed (alternated) after $R_{1}$ by the goldfish's swimming from one end to the other via the response-contingent programming. Shock was scheduled to occur at 12, 14, and $15 \mathrm{sec}$ after trial onset. Shock was omitted only if the relationship between the color of the center trial standard and the color of the response-controlled ends met the appropriate criterion. For the matching procedure, the criterion required the center and end lights to be the same color. For the oddity procedure, the criterion required that the center and end lights be different colors. The instrumentation tested the relationship between center trial standard and end colors at each of the scheduled shock times and administered shock accordingly. At the end of the last shock $(15.2 \mathrm{sec})$, all trial colors were terminated and the white ITI illumination was reestablished.

The purpose of the three-shock procedure was twofold. First, an animal that did not respond until the first shock at $12 \mathrm{sec}$ could then shuttle, and assuming this "shock-elicited" initial response produced the proper relationship between center and end colors, further shocks would be omitted (or escaped). If, because the initial "shock-elicited" response produced the wrong color relationship and a second shock was administered, another response could change the relationship and the third shock would be omitted. In effect, the three-shock procedure was a kind of correction procedure that allowed the goldfish to prevent further shock on trials in which initial errors occurred. The second purpose of the three-shock procedure was to minimize crediting random multiple responses as avoidances. The random-multipleresponding animal would, on average, miss the first shock at $12 \mathrm{sec}$ on half of the trials when it responded before the first shock. But if it continued to respond, the end colors would alternate with each response, which necessarily changed the criterion relationship between end and center trial standard. The probability of a randomly responding animal's missing all three shocks by chance was considerably less than $50 \%$. Staggering the intershock intervals aided in detecting the randomly responding animal as well. To be credited with an avoidance, the goldfish had to respond initially $\left(R_{1}\right)$ to 
turn on the end lights, and then, if necessary, respond again $\left(\mathbf{R}_{\mathbf{c}}\right)$ to adjust the end colors so that the relationship between the current center trial standard and the response-contingent end colors met the appropriate procedural criterion at all three scheduled shock times. Thus, missing the first shock did not mean that all further shocks were omitted if the animal continued to respond.

Original acquisition. The first 30 days of training constituted the original acquisition phase. Half of the goldfish $(n=12)$ were trained with the matching procedural criterion, and the remaining half $(n=12)$ with the oddity procedural criterion. With each procedure, half $(n=6)$ had red/green signal colors and the remainder $(n=6)$ had blue/yellow signal colors. This constituted a factorial arrangement of the two procedural types (matching, oddity) and signal colors (red/green and blue/yellow), yielding four different criterion $\times$ color groups.

Extinction. In extinction testing, half $(n=3)$ of each criterion $x$ color group had the signal colors changed (e.g., red/green to blue/yellow, or vice versa), and the remaining half $(n=3)$ were extinguished with the same signal colors experienced in original acquisition. The extinction procedure differed from that used in acquisition in that the goldfish were no longer required to choose between the signal colors to avoid shock. However, the animals were required to initially respond. Specifically, animals were still required to make $R_{1}$, but not $R_{c}$, to avoid shock. Thus, all shocks were omitted following the goldfish's first response of a trial, regardless of the signal color resulting from that or subsequent responses. If the first response occurred before $12 \mathrm{sec}$, all shocks were omitted. If one or more shocks were administered and elicited a response, all further shocks were omitted. Essentially, the $\mathbf{R}_{\mathbf{1}}$ response requirement was retained, but the $\mathbf{R}_{c}$, or choice, requirement was dropped. All animals were tested for 30 days in the extinction phase.

Reacquisition. In reacquisition, all animals were returned to their respective original-learning color-signal conditions and tested for an additional $\mathbf{3 0}$ days with colors and procedures identical to those of original acquisition. Reacquisition was simply replacing the $\mathbf{R}_{\mathbf{c}}$ criterion to avoid shock, which had been eliminated during the extinction phase, thus returning everything to the conditions of original acquisition.

Measures. At the end of each day's training, for each of the 24 goldfish, three items were recorded: the number of trials with at least one response before the first shock (TwR), the number of trials when the procedural criterion was met at the time scheduled for the first shock $\left(A_{1}\right)$, and the number of trials when the procedural criterion was met for all three scheduled shock times (A). Using these measures, two other performance measures, discrimination indexes (DI), for the $A_{1}$ and $A$ measures were calculated. The DI measure was $[(S+)-(S-)] / 40$, where $S+$ is the number of trials in which the animal responds $\left(R_{1}\right)$ and chooses $\left(R_{c}\right)$ the correct color relationship and meets the procedural criterion and thus avoids, where $S-$ is the number of trials in which the animal responds $\left(R_{1}\right)$ but chooses $\left(R_{c}\right)$ the wrong color relationship and thus does not meet the procedural criterion, and where $S_{+}$and $S_{-}$, of course, sum to TwR. The DI measure has limits of \pm 1.00 , where 1.00 means the animal discriminates perfectly on all trials, 0.00 indicates the animal performs at chance discrimination levels, regardless of the number of trials with response, and -1.00 indicates a perfect negative discrimination on all trials. Furthermore, since the denominator is 40 , the DI differentiates between animals responding on a few trials and those responding on many or all trials. DI measures were calculated for the $A_{1}\left(D_{1}\right)$ and $A\left(D I_{3}\right)$ measures.

Some explanation of the $A$ and $A_{1}$ as measured during extinction must be made. During extinction, the animal was required to respond $\left(R_{1}\right)$, but the choice contingency $\left(R_{c}\right)$ was suspended. The $A$ and $A_{1}$ measures were used in original acquisition and reacquisition as $R_{c}$ measures. During extinction, the $R_{c}, A_{1}$, and $A$ measures were retained, but indicated the number of trials in which the animal would have met the acquisition $R_{c}$ procedural criterion if the acquisition $\mathbf{R}_{c}$ procedural criterion had been in force.

\section{RESULTS}

All figures present data in 10 3-day blocks. Data were analyzed both over 30 days and in 10 3-day blocks, but no essential differences were observed and only the 10 3-day block analyses for each phase are presented. All analyses were repeated measures ANOVAs with procedural criterion (matching vs. oddity) and same or changed (in extinction) signal colors as independent dimensions, and 10 3-day block as the repeated dimension. Appropriate partitions were calculated when required to facilitate interpretation of the data.

\section{Original Acquistion}

Figure 1 shows the average trials with response (TwR), avoidances (A), and first shock avoidances $\left(A_{1}\right)$ for each of the four groups defined by the extinction conditions. In addition, for comparison purposes only, an expected avoidance measure, $\mathrm{E}$ (avoid), is also incorporated in the figure. The $\mathrm{E}$ (avoid) measure is determined by halving the daily TwR performance $(.50 \times \mathrm{TwR})$, and represents a chance expectancy for the avoidance performance. From Figure 1, it is clear that all groups increase their performance with training. Groups show progressive increases in trials with responses (TwR or $R_{1}$ ),

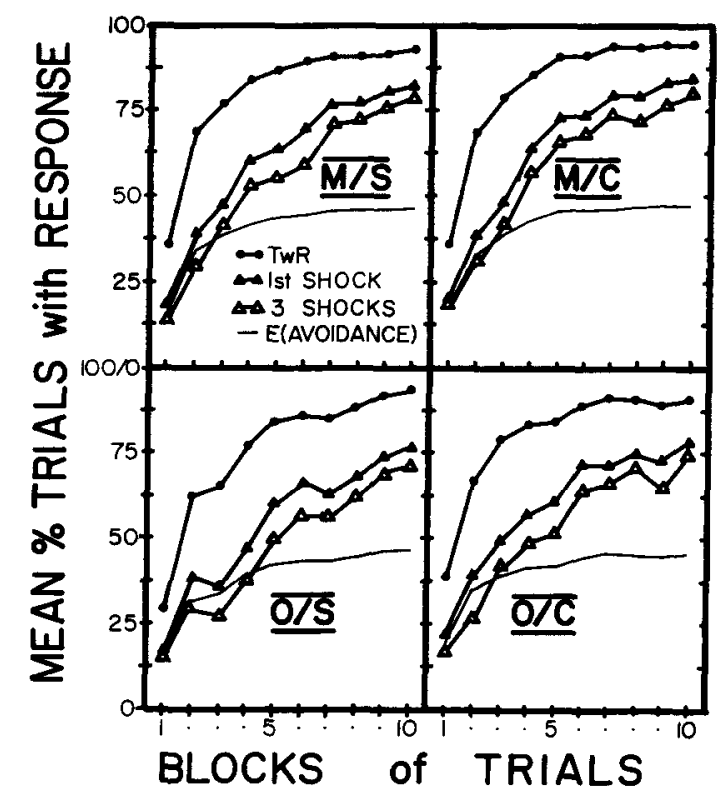

Figure 1. The mean percent trials with response ( $T w R$ ), trials in which the first shock of a trial was omitted (first shock), and trials in which all three shocks were omitted (three shocks), in 3-day blocks (120 trials) for the 30 days of the original acquisition phase. Shown are performances for the matching same (M/S), matching changed $(M / C)$, oddity same $(O / S)$, and oddity changed $(O / C)$ color groups as they were configured in the next or extinction phase. In addition, chance avoldance measure, $\mathrm{E}$ (avoidance), is included, where $E$ (avoldance) is half of $T w R(.5 \times T w R)$, for comparison purposes only. 
avoiding the first shock at $12 \sec \left(A_{1}\right)$, and avoiding all three shocks (A). Both the $A_{1}$ and $A$ avoidance measures are clearly well above a $50 \%$ chance expectancy by the middle of original acquisition training, indicating that all animals learned to choose $\left(\mathbf{R}_{c}\right)$ the appropriate end color (matching or oddity) with training. It is also clear that the respective halves of each criterion procedure designated for same or changed colors in extinction did not differ in original acquisition. The only difference found was that the matching groups learned faster and achieved slightly higher conditional discrimination performance levels than the oddity groups. All these impressions were supported by the analyses. For the TwR analyses, only a significant increase with training over blocks was observed $[F(9,180)=250.21, p<.01]$. For the A avoidance measure, a significant increase with training over blocks $[F(9,180)=192.47, \mathrm{p}<.01]$ and a significant difference between the matching and oddity procedures $[\mathrm{F}(1,20)=5.26, \mathrm{p}<.05]$ were observed. On the $A_{1}$ measure, significant increases with training over blocks $[F(9,180)=251.23, p<.01]$ and matching/oddity differences $[F(1,20)=5.23, p<.05]$ were observed, but an interaction between matching/oddity and blocks was also seen $[F(9,180)=$ $2.65, \mathrm{p}<.01$ ]. A partition over the last five blocks showed that this interaction had vanished $[F(4,180)$ $=1.69$ ] by the end of training, and thus was restricted to the initial phases of acquisition only. In addition to the above measures, analyses for the two derived discrimination index (DI) measures were also calculated. The average DI values for 3-day blocks for the $A_{1}$ measure $\left(D_{1}\right)$ and $A$ or avoidance measures $\left(\mathrm{DI}_{3}\right)$ for all groups are shown in Figure 2. Figure 2 shows not only that all groups increase their performance levels on both the $\mathrm{DI}_{1}$ and $\mathrm{DI}_{3}$ measures, but that they also learn to conditionally discriminate in the matching and oddity procedural criterion conditions. This is easy to see, since a .01 chance envelope is incorporated in the figure, and all groups conditionally discriminate significantly above a zero or chance level by the fifth 3-day block of original acquisition on both the $\mathrm{DI}_{1}$ and $\mathrm{DI}_{3}$ measures. As with the other measures, it is clear that all groups learn, that there are no differences between halves of each criterion group designated to be extinguished with the same or changed colors, and that the matching groups are slightly superior to the oddity groups. Analyses confirmed each of these impressions. For the $\mathrm{DI}_{3}$ measure, which compared discriminations based on avoiding all three shocks per trial, all groups showed significant increases over training blocks $[\mathrm{F}(9,180)=100.81, \mathrm{p}<.01]$, the matching groups were superior to the oddity groups $[F(1,20)=5.16$, $\mathrm{p}<.05]$, and an interaction was observed between procedure (matching/oddity) and training blocks $[F(9,180)=2.41, p<.05]$. However, this interaction vanished over the last five blocks of training

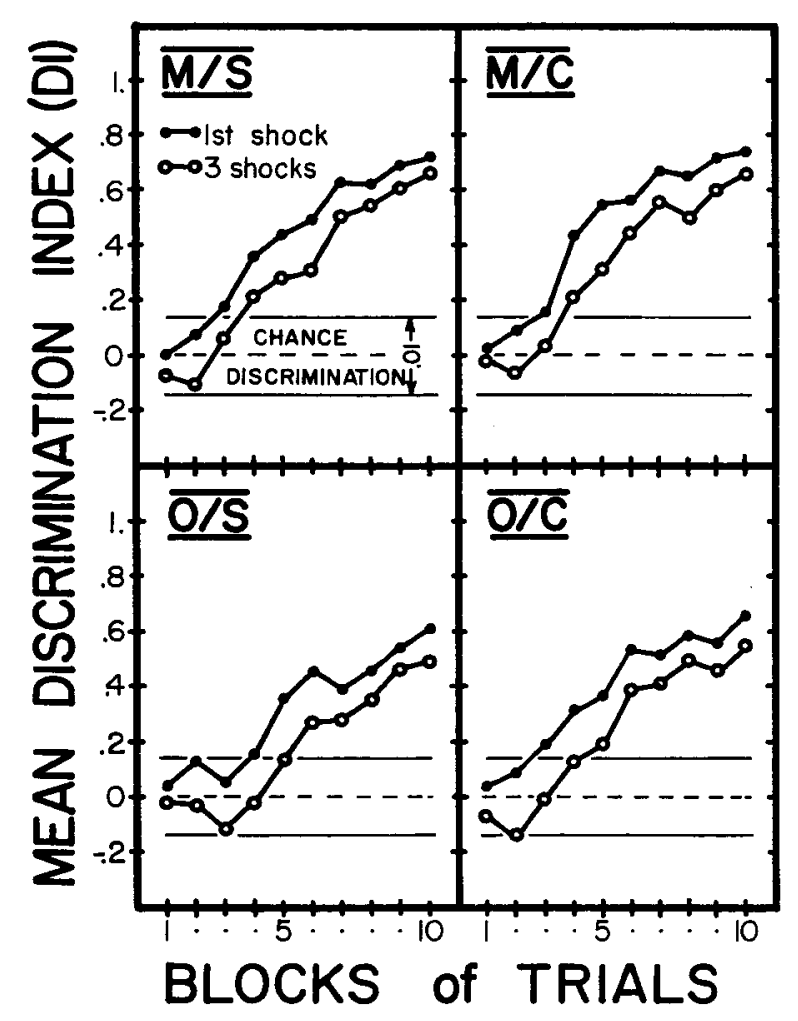

Figure 2. The mean by 3-day block (120 trials) discrimination indexes (DI) for missing the first shock of a trial, and all three shocks on a trial for the $\mathbf{3 0}$ days of original acquisition are shown. The matching same $(M / S)$, matching changed $(M / C)$, oddity same $(O / S)$, and oddity changed $(O / C)$ color groups, as they were configured in the next or extinction phase, are shown. In addition, a .01 chance discrimination envelope about zero or chance discrimination is incorporated in the figure. Any performance outside of this envelope indicates different-from-chance conditional discrimination performance.

$[F(4,180)=1.29]$. For the $\mathrm{DI}_{1}$ measure, which compared discriminations only for the first shock at $12 \mathrm{sec}$, groups showed significant improvement over training blocks $[F(9,180)=153.62, p<.01]$ and the matching groups were superior to the oddity groups $[F(1,20)=6.95, p<.01]$. A matching/oddity $\times$ blocks interaction was also observed over the 10 training blocks $[F(9,180)=4.38, p<.01]$ and, although attenuated, was still present over the last five blocks $[F(4,180)=2.82, p<.05]$. However, this interaction was not present on the last training block of original acquisition $[F(1,180)=.86]$.

There is a certain amount of redundancy in Figures 1 and 2 . One can argue that the $A_{1}$ and $A$ measures show the same information as the $\mathrm{DI}_{1}$ and $\mathrm{DI}_{3}$ measures. To some extent this is true. However, the DI measures allow one to incorporate chance conditional discrimination criterion levels into the figure. This allows one to see when actual performances exceed chance discrimination levels by inspection. Because of this, only the DI measures will be used to 
present the performance in the remaining two phases of the experiment.

\section{Extinction}

The first item of interest in extinction is whether or not goldfish continue to "perform." Recall that, with pigeons, extinction produces large decrements in keypeck response strength. The comparable measure with goldfish is the TwR or $R_{1}$ performance measure. In the present experiment, goldfish were responding on $93 \%$ of their trials at the end of the original acquisition phase. On the first block of extinction testing, TwR performance dropped to $90 \%$ of the trials, but then increased, so that from the fourth block on, they responded on $95 \%$ of their trials. The TwR analysis confirmed this by showing a slight, but significant, increase in TwR over the 10 3-day blocks of extinction $[F(9,180)=9.80, p<.01]$. No other differences on the TwR measure were observed. Clearly the $R_{1}$ extinction procedure had the desired effect: Goldfish maintained or even improved their $R_{1}(T w R)$ performance during the extinction phase.

Conditional discrimination performances during the extinction phase are shown in Figure 3. Figure 3 shows that conditional discrimination performances dropped progressively and rapidly to chance (or below) for all four groups. If one compares the left (same) and right (changed) panels, it is also clear that groups with their signal colors changed from those experienced in original acquisition dropped faster and leveled out lower on the DI measures than those groups extinguished with the same signal colors they had had in original acquisition. This is evident for the measures showing avoidance for the first shock $\left(\mathrm{DI}_{1}\right)$ and all three shocks $\left(\mathrm{DI}_{3}\right)$ as well. Note that conditional discrimination performance for the changed color groups (both right panels) drops below chance discrimination performance and, in fact, deviates from chance or zero DI significantly (below -.14 DI). This is especially evident in the oddity/changed $[\mathrm{O} / \mathrm{C}]$ group's performance (lower right panel). However, note that on the $\mathrm{DI}_{1}$ measure, which indicates missing the first shock only, conditional discrimination performances stay within the .01 chance discrimination envelope. From the differences in these two measures, it is clear that goldfish in the changed signal color groups tend to respond more often during the intershock interval than those retaining the same signal colors. It also implies that the $\mathrm{DI}_{3}$ measure, which is based on a $50 \%$ chance discrimination expectancy, is a conservative estimate or overestimate of chance discrimination performance.

The bulk of these impressions were confirmed by the analyses. For the $\mathrm{DI}_{3}$ measure, significant drops in discrimination were observed over blocks $[F(9,180)$ $=14.16, p<.01]$, and changed color groups were found to extinguish faster than the same color groups $[F(1,20)=8.89, p<.01]$. In addition, a matching/

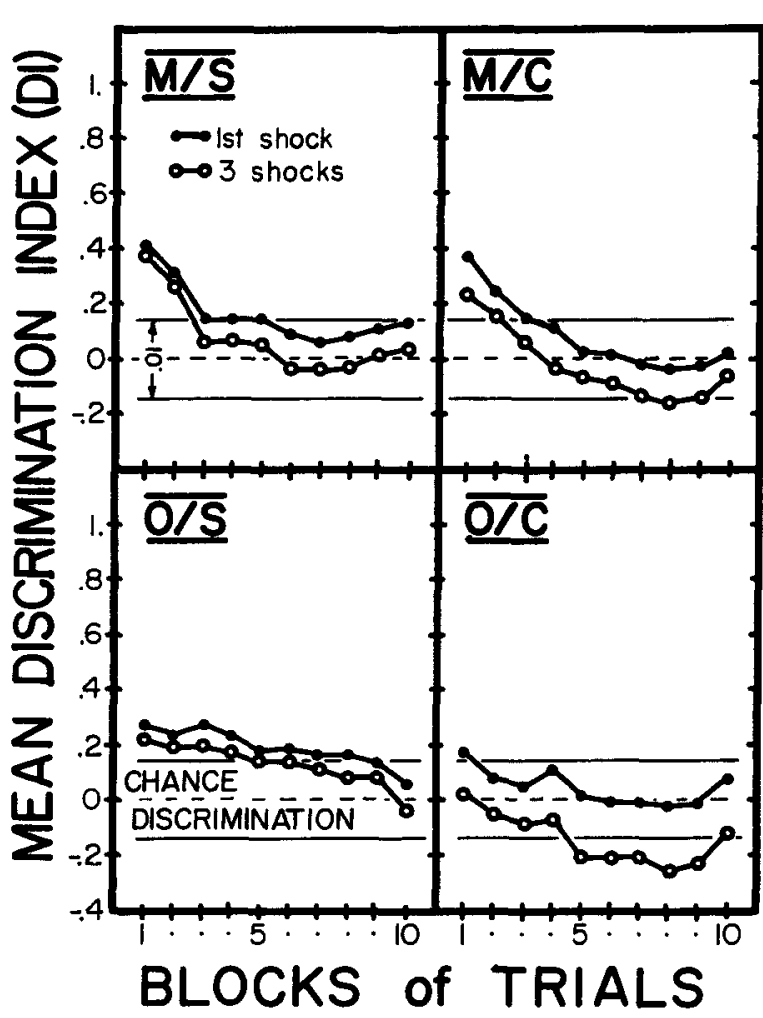

Figure 3. The mean 3-day block (120 times) discrimination indexes for avoiding the first shock $\left(A_{1}\right)$ and avolding all three shocks (A) for the matching/same $(M / S)$, matching/changed (M/C), oddity/same $(O / S)$, and oddity/changed $(O / C)$ color groups for the 30 days of extinction. Note that these do not indicate actual avoidances, but what would have happened if the acquisition avoidance criterions had been in effect. $\mathbf{A} .01$ chance discrimination envelope showing the range of "chance discrimination" on the DI measures is included. DI performance outside of this envelope indicates different-from-chance performance.

oddity $\times$ blocks interaction was observed $[F(9,180)=$ $2.03, \mathrm{p}<.05]$. A partition of this interaction showed that only the oddity same and changed groups differed in extinction $[F(1,10)=10.86, p<.01]$. The matching changed and same signal color groups did not $[\mathrm{F}=1.11]$.

The analyses and partitions for the $\mathrm{DI}_{1}$ and avoidance measures generated the same pattern of significant differences and thus are omitted for brevity. From the present results, it is clear that the feature of the goldfish's performance that extinguishes is their "choice" $\left(\mathbf{R}_{\mathrm{c}}\right)$ of the matching or oddity signal color. This occurs because they maintain their $R_{1}$ (TwR) performances at or above those achieved in original acquisition. Thus, the present data clearly show that matching and oddity conditional discriminations $\left(\mathbf{R}_{c}\right)$ will extinguish when differential reinforcement for choice is removed or equated.

\section{Reacquisition}

Following extinction testing, all groups had their signal conditions returned to those experienced in 
original learning. Procedurally, the reacquisition phase was identical to the original acquisition phase. The results for the $\mathrm{DI}_{1}$ and $\mathrm{DI}_{3}$ measures are shown for all four groups in Figure 4. Figure 4 shows that with the conditional discrimination differential reinforcement replaced $\left(\boldsymbol{R}_{\mathrm{c}}\right)$, all animals in all groups reacquired the conditional discrimination much faster than in original acquisition. Note that all groups display conditional discrimination performance above chance levels, on both the $\mathrm{DI}_{3}$ and $\mathrm{DI}_{1}$ measures, by the second 3-day block of reacquisition training. In contrast, comparable conditional discrimination performance levels were not reached by all groups on both measures in original acquisition until the fifth 3-day block (see Figure 2), although some groups reached this level as early as the fourth block. These impressions were confirmed by the analyses. The first analysis was on the TwR measure. As in extinction, no differences were found between groups. Furthermore, there was no change in TwR performance levels over the 10 3day blocks of reacquisition in TwR $(F=1.06)$. Goldfish responded on about $91 \%$ of all trials in this last

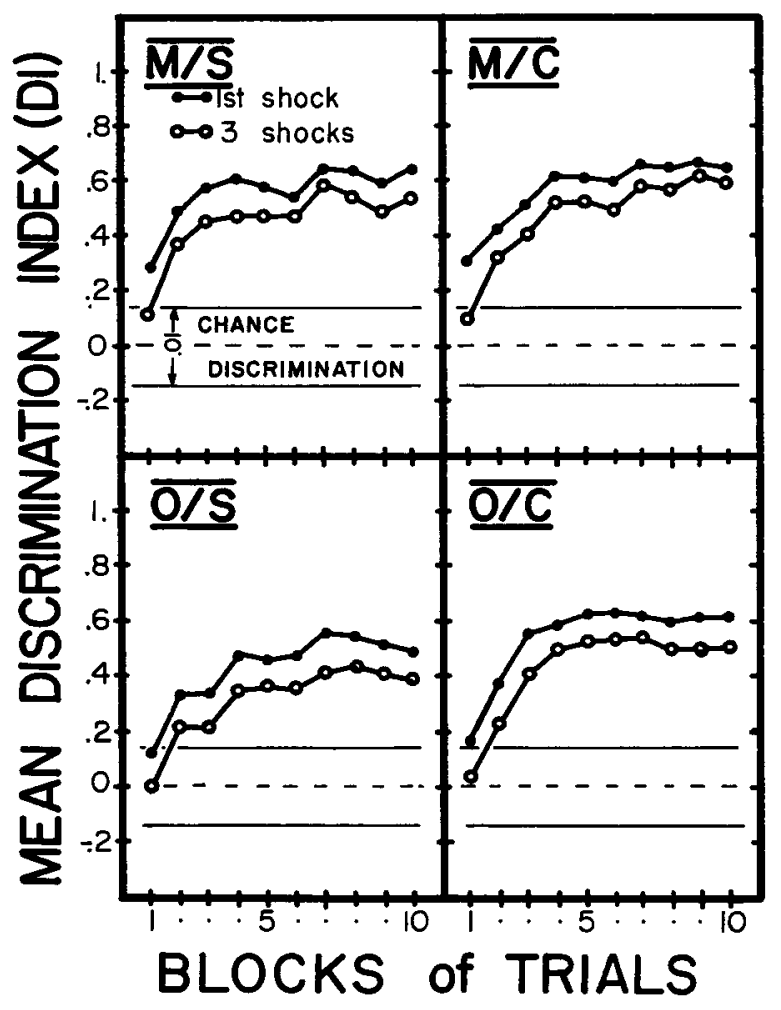

Figure 4. The mean 3-day block (120 trials) discrimination indexes for avolding the first shock $\left(A_{1}\right)$ and avoiding all three shocks (A) for the matching/same (M/S), matching/changed $(\mathrm{M} / \mathrm{C})$, oddity/same $(\mathrm{O} / \mathrm{S})$, and oddity/changed $(\mathrm{O} / \mathrm{C})$ color groups for the 30 days of reacquisition. $A$.01 chance discrimination envelope showing the range of chance discrimination on the DI measure is included. Any DI performance outside of this envelope differs from chance level discrimination. phase. On the $\mathrm{DI}_{3}$ measure, no group differences were observed. Only an increase in conditional discrimination on the $\mathrm{DI}_{3}$ measure was observed $[F(9,180)=39.66, p<.01]$. Slight differences between matched and oddity criterion procedures were found on the DI $\mathrm{D}_{1}$ measure $[\mathrm{F}(1,20)=5.89, \mathrm{p}<.01]$ with matching superior to oddity, comparable to the finding in original acquisition. All groups also showed dramatic increases in conditional discrimination on the $\mathrm{DI}_{1}$ measure $[\mathrm{F}(9,180)=32.03, \mathrm{p}<.01]$. Comparisons of acquisition and reacquisition DI performances found reacquisition DI performances higher on Blocks $2,3,4$, and $5[\operatorname{ts}(180)=15.12$, $15.04,12.57$, and 8.90 , all ps $<.01$, respectively], but not statistically different on Blocks $1,6,7,8,9$, and 10. Other analyses produced results comparable to the $\mathrm{DI}_{1}$ and $\mathrm{DI}_{3}$ measures and are omitted for brevity.

\section{DISCUSSION}

The present results confirm and extend Zerbolio and Royalty's (1983) finding of conditional discrimnation learning in goldfish. Acquisition confirms the finding that goldfish can learn such a difficult discrimination. The only difference is that matchingtrained goldfish were slightly superior in the present study, whereas oddity-trained goldfish were slightly superior in the earlier study. However, study-tostudy changes in matching/oddity superiority are found with other animals as well (Zentall \& Hogan, $1974,1978)$. It is not obvious why this occurs.

The extinction procedure functioned as expected. All goldfish showed rapid and progressive decrements in choice accuracy so that by the end of the extinction phase, all were discriminating at chance matching or oddity choice levels. Throughout the extinction phase, TwR or performance levels remained at or above those achieved in acquisition. Clearly, the extinction procedure diminished the accuracy of the choice response without affecting performance levels.

Although all goldfish extinguished on the accuracy measure, one would expect that groups with their signal color changed would suffer some generalization decrement and extinguished faster than those tested with the same signal colors experienced in acquisition. Partial support for this was found. The oddity-trained changed-color-signal groups extinguished significantly faster than the same-colorsignal groups. No differences were observed for the changed/same signal-color matching-trained groups. What is clear is that the accuracy of the conditional matching or oddity choice extinguished in all groups regardless of signal colors of matching/oddity criterion by the end of extinction testing.

The critical phase for the conceptual generalization of extinction prediction is the reacquisition phase. In reacquisition, all groups were tested with the colors 
they had experienced in original acquisition. Although the matching groups were slightly superior to the oddity groups in reacquisition, just as they were in acquisition, no differences between same and changed signal-color group halves of the matching/oddity criterion procedures were observed in reacquisition. Clearly extinction training with new or changed signal colors was jusi as effective in diminishing the matching or oddity choice response as extinction training with the original acquisition signal colors. Since one would expect generalization of extinction effects via the matching or oddity concept to be independent of signal color, these results support the interpretation that goldfish not only learn a conditional discrimination at the conceptual level, but extinguish a conditional discrimination at the conceptual level as well.

One of the curious facets of the present study is that the extinction results are precisely opposite to those found earlier. With pigeons, positive reinforcement, and an extinction procedure of turning of the food dispenser, response strength (comparable to TwR) decremented, but the accuracy of the conditional discrimination choice response remained intact (Cohen, 1969; Cumming et al., 1967; Looney et al., 1977; Lydersen \& Cheney, 1973). In the present study, TwR remained intact but choice accuracy decremented. This comparison of positive and negative reinforcement extinction results poses an interesting reversal of the usual extinction expectation. Negatively reinforced responses are expected to be more resistant to extinction than positively reinforced responses. In the conditional discrimination design, the positively reinforced matching/oddity choice response appears more resistant to extinction that the negatively reinforced choice response. However, this difference is clearly procedural. In acquisition, both for the pigeon and goldfish, an initial response $\left(R_{1}\right)$ is necessary to allow access to the signals representing different choices. If no $R_{1}$ occurs, the different choice signals are not presented, and a choice response $\left(\mathbf{R}_{c}\right)$, de facto, cannot occur. However, both pigeons and goldfish learn the $R_{1}$ response quickly. Once $R_{1}$ is established, exposure to the different choice signals occurs regularly, and exposure to the consequences of differential choice between them can occur. Both pigeons and goldfish learn to choose $\left(\mathbf{R}_{\mathrm{c}}\right)$ the specific signal paired with their preferred level of reinforcement. Now consider what occurs in extinction. Keep in mind that one interpretation of extinction is that it represents a procedure designed to inform the animal of a change in the reinforcement contingencies from those present in acquisition (Bolles, Moot, \& Grossen, 1971; Davenport \& Olson, 1968; Mackintosh, 1974). With goldfish, the $R_{1}$ response is forced because the $R_{1}$ shockomission contingency remains in force. Since $R_{1}$ is forced, $R_{c}$ is also forced, because $R_{1}$ necessarily implies an $\mathbf{R}_{c}$ response. Since $\mathbf{R}_{c}$ occurs, the goldfish can be exposed to the change in differential $R_{c}$ consequences. As a result, $R_{c}$ accuracy diminishes because all $\mathbf{R}_{c}$ responses lead to equivalent consequences in extinction. This does not occur with pigeons when the traditional extinction procedure of turning off the food dispenser is used. With the food dispenser turned off, all responses, $\mathbf{R}_{\mathbf{1}}$ and $\mathbf{R}_{c}$, lead to negative reinforcement. The pigeon quickly diminishes its response strength, but this means it decrements on the $R_{1}$ response. Without $R_{1}$, the different $\mathbf{R}_{c}$ signals are not presented and no $\mathbf{R}_{c}$ choice response is possible. Essentially, diminishing $R_{\mathbf{1}}$ response strength limits the number of occasions when $\mathbf{R}_{\mathrm{c}}$ is possible, and therefore limits exposure to the change in differential reinforcement contingencies associated with each of the $\mathbf{R}_{\mathrm{c}}$ responses. If extinction provides only limited exposure to the change in $R_{c}$ response contingencies, it is not surprising that the $\mathbf{R}_{c}$ response remains intact. Pigeons do not extinguish on the $\mathbf{R}_{c}$ accuracy measure because the $\mathbf{R}_{c}$ response has not been subjected to many extinction trials. A slight alteration in the pigeon extinction procedure should provide a test of this interpretation. If extinction were to consist of reinforcing all $\mathbf{R}_{c}$ responses with food, they should continue to respond $\left(R_{1}\right)$ but their $\mathbf{R}_{\mathrm{c}}$ accuracy should decrement. If this occurs, testing for conceptual generalization of extinction with pigeons and positive reinforcement procedures would be possible.

In sum, the present results confirm that goldfish learn to choose the matching or odd color in a conditional discrimination procedure to avoid shock. Furthermore, in extinction, with a procedure designed specifically to extinguish the choice response, the matching or oddity choice response decrements to chance discrimination levels. In addition, regardless of whether the signal colors used in extinction are those experienced in original acquisition or are new colors, extinction generalizes to other signal colors so that all extinguished groups start at the same chance discrimination levels and reacquire at the same speed in a reacquisition test. The present extinction results, along with the earlier transfer results shown by Zerbolio and Royalty (1983), are consistent with the interpretation that goldfish learn conditional discrimination problems to avoid shock at the conceptual level.

\section{REFERENCES}

Bolles, R. C., Moot, S. A., \& Grossen, N. E. (1971). The extinction of shuttlebox avoidance. Learning and Motivation, 2, 324-333.

Cohen, L. R. (1969). Generalization during acquisition, extinction, and transfer of matching with an adjustable comparison. Journal of the Experimental Analysis of Behavior, 12, $463-474$.

Cumming, W. W., Berryman, R., Cohen, L. R., \& Lanson, R. N. (1967). Some observations on extinction of a complex discriminated operant. Psychological Reports, 20, 1328-1330. 
Davenport, D. E., \& Olson, R. D. (1968). A reinterpretation of extinction in discriminated avoidance. Psychonomic Science, 13, 5-6.

Looney, T. A., Cohen, I. R., Brady, J. H., \& Cohen, P. S. (1977). Conditional discrimination performance by pigeons on a response-independent procedure. Journal of the Experimental Analysis of Behavior, 27, 363-370.

Lydersen, T., \& Cheney, C. D. (1973). Fixed-ratio discrimination effects of extinction and satiation. Journal of Biological Psychology, 15, 26-29.

MACKInTOSH, N. J. (1974). The psychology of animal learning. London: Academic Press.

Zentall, T. R., \& Hogan, D. E. (1974). Abstract concept learning in the pigeon. Journal of Experimental Psychology, 102, 393-398.
Zentall, T. R., \& Hogan, E. (1978). Same/different concept learning in the pigeon: The effect of negative instance and prior adaptation to transfer stimuli. Journal of the Experimental Analysis of Behavior, 30, 177-186.

Zerbolio, D. J., \& Royalty, J. L. (1982). Acquisition and extinction of differential responses to signais paired with shock or shock-omission in goldfish: Evidence for truly discriminated avoidance learning. Animal Learning \& Behavior, 10, 377-389.

Zerbolio, D. J., \& RoYAlty, J. L. (1983). Matching and oddity conditional discrimination in the goldfish as avoidance responses: Evidence for conceptual avoidance learning. Animal Learning \& Behavior, 11, 341-348.

(Manuscript received October 28, 1983; revision accepted for publication March 29, 1984.)

\title{
ANNOUNCEMENT \\ 25th Annual Meeting of The Psychonomic Society, Inc. Change of Dates for Meeting
}

The dates for the 25th annual meeting of The Psychonomic Society, Inc., have been changed from November 1-3, 1984, to:

November 8-10, 1984.

The meeting still will be held at the Hilton Palacio Del Rio, in San Antoni, Texas.

\section{NOTICE}

\section{Search for Editor of Memory \& Cognition}

Nominations are being solicited for the editorship of Memory \& Cognition. The new editor is expected to begin handling submissions about January 1, 1985, or shortly thereafter. Nominations of qualified individuals should be sent, by August 1, 1984, to:

\author{
Robert G. Crowder \\ Department of Psychology \\ Yale University \\ Box 11A Yale Station \\ New Haven, CT 06520
}

